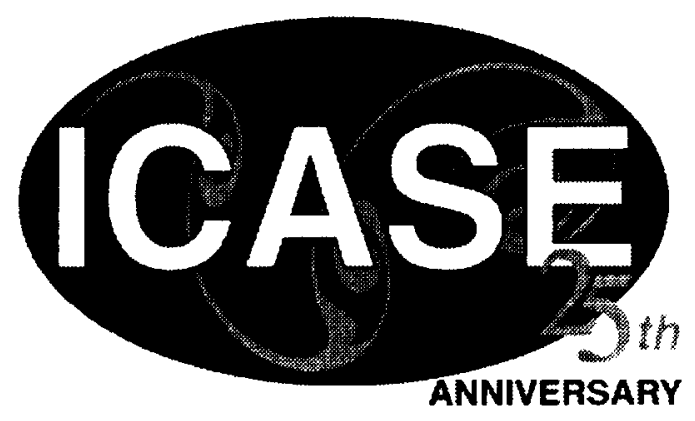

\title{
COMPARISON OF SEVERAL DISSIPATION ALGORITHMS FOR CENTRAL DIFFERENCE SCHEMES
}

\author{
R. C. Swanson \\ R. Radespiel \\ E. Turkel
}

NASA Contract No. NAS1-19480 August 1997

Institute for Computer Applications in Science and Engineering NASA Langley Research Center

Hampton, VA 23681-0001

Operated by Universities Space Research Association

Nest

National Aeronautics and

Space Administration

Langley Research Center

Hampton, Virginia 23681-0001 


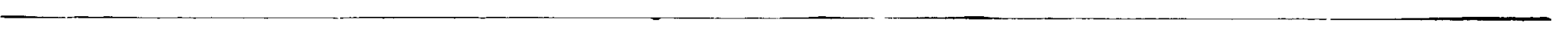

'

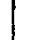




\title{
COMPARISON OF SEVERAL DISSIPATION ALGORITHMS FOR CENTRAL DIFFERENCE SCHEMES
}

\author{
R. C. Swanson \\ Aerodynamic and Acoustic Methods Branch \\ NASA Langley Research Center \\ Hampton, VA \\ R. Radespiel, \\ DLR, Institute of Design Aerodynamics \\ Braunschweig, Germany \\ E. Turkel \\ ICASE, NASA Langley \\ and Department of Mathematics \\ Tel-Aviv University, Tel-Aviv, Israel *
}

\begin{abstract}
Several algorithms for introducing artificial dissipation into a central difference approximation to the Euler and Navier Stokes equations are considered. The focus of the paper is on the convective upwind and split pressure (CUSP) scheme, which is designed to support single interior point discrete shock waves. This scheme is analyzed and compared in detail with scalar and matrix dissipation $(M A T D)$ schemes. Resolution capability is determined by solving subsonic, transonic, and hypersonic flow problems. A finite-volume discretization and a multistage time-stepping scheme with multigrid are used to compute solutions to the flow equations. Numerical results are also compared with either theoretical solutions or experimental data. For transonic airfoil flows the best accuracy on coarse meshes for aerodynamic coefficients is obtained with a simple MATD scheme.
\end{abstract}

\footnotetext{
*This research was supported in part by the National Aeronautics and Space Administration under NASA Contract No. NAS1-19480 while the first author was in residence at the Institute for Computer Applications in Science and Engineering (ICASE), NASA Langley Research Center, Hampton, VA 23681-0001.
} 



\section{Introduction}

Accuracy must be a primary consideration in the construction of any numerical scheme. One would like to devise a scheme with the minimum amount of artificial dissipation required for stability, as well as convergence in the case of a stationary solution. For fluid dynamic computations the numerical scheme should be designed to have high accuracy in smooth regions of the flow field and well resolved shock waves and contact discontinuities. According to Harten [3] such discrete formulations, where the accuracy away from discontinuities is at least second order, are called high resolution schemes. The design of these schemes for systems of conservation laws is generally based on theory developed for a scalar conservation law. As a consequence one cannot ensure that the properties of the scheme for the scalar equation are valid for the system. In addition, schemes that permit high definition of shock waves without oscillations are first order in the neighborhood of shocks. Concern naturally arises regarding contamination of the solution, especially in the case of viscous flows. For these reasons the properties and resolution capability of this class of schemes must be verified through numerical applications for a wide range of flow conditions.

High resolution schemes of particular interest for solving the compressible fluid equations are those that allow shock capturing with a single interior point. In Ref. [6] Jameson presents the convective upwind and split pressure (CUSP) scheme. For this scheme the artificial diffusive flux vector associated with a given coordinate direction is expressed in terms of changes in the state and flux vectors. A somewhat limited number of inviscid and viscous computations have been performed to evaluate these schemes (see Refs. $[6,7]$ and $[22,23,9]$ ).

In the current work we investigate and analyze the HCUSP version [6] of the CUSP scheme. The HCUSP scheme allows a solution with constant total enthalpy for steady flow. We discuss the shock-capturing behavior for various choices of the dissipation coefficients. We introduce a simple modification of the limiter function, which is generally used with the scheme, to control background dissipation, and thus global accuracy. The HCUSP scheme includes a contribution that is scaled according to the local velocity. If the velocity approaches zero, as it does for viscous flow near a solid boundary, and there is a high aspect ratio mesh, the dissipation in the streamwise direction (i.e., direction of long side of mesh cell) may not be adequate for convergence. A change in the velocity scaling factor based on aspect ratio is presented. The resolution capability of the HCUSP scheme is evaluated for subsonic, transonic, and hypersonic flow problems. A detailed comparison of the scheme with a scalar and matrix dissipation schemes is performed. The scalar scheme is based on the dissipation model of Jameson, Schmidt, and Turkel [4] and is often used with central differencing.

\section{Dissipation}

A finite-volume approach is applied to discretize the fluid dynamic equations of motion. The computational domain is divided into quadrilateral cells, fixed in time, and for each cell the governing equations can be nondimensionalized and written in integral form as follows:

$$
\frac{\partial}{\partial t} \iint_{\Omega} w d x d y+\int_{\partial \Omega}(f d y-g d x)=\frac{\sqrt{\gamma} M}{R e} \int_{\partial \Omega}\left(f_{v} d y-g_{v} d x\right)
$$

where $\Omega$ is a generic cell with boundary $\partial \Omega$. In the scaling factor for the viscous terms on the right hand side of Eq. (1), the quantities $\gamma, M$, and $R e$ are the ratio of specific heats, Mach number, and Reynolds number, respectively, with $M$ and $R e$ defined in terms of nominal conditions. Taking $w_{j, k}$ as the cell-averaged solution vector, Eq. (1) can be written in semidiscrete 
form as

$$
\frac{d}{d t}\left(\Omega_{j, k} w_{j, k}\right)+\mathcal{L} w_{j, k}=0
$$

where $\Omega_{j, k}$ is the area of the cell, and $\mathcal{L}$ is defined by

$$
\mathcal{L}=\mathcal{L}_{C}+\mathcal{L}_{D}+\mathcal{L}_{A D}
$$

with the subscripts $C, D$, and $A D$ referring to convection, diffusion, and artificial dissipation. In order to simplify the description of the dissipation models, we consider the one-dimensional Euler equations of gas dynamics.

\section{Scalar Dissipation Model}

The scalar dissipation is based on the model introduced by Jameson, Schmidt, and Turkel [4]. This model defines a switching function based on a blending of the second and fourth differences. The term associated with the operator $\mathcal{L}_{A D}$ is expressed as

$$
\mathcal{L}_{A D} w_{j}=-\left(D^{2}-D^{4}\right) w_{j}=d_{j+1 / 2}-d_{j-1 / 2}
$$

Then

$$
\begin{aligned}
D^{2} w_{j} & =\nabla\left[\left(\lambda_{j+1 / 2} \varepsilon_{j+1 / 2}^{(2)}\right) \Delta\right] w_{j}, \\
D^{4} w_{j} & =\nabla\left[\left(\lambda_{j+1 / 2} \varepsilon_{j+1 / 2}^{(4)}\right) \Delta \nabla \Delta\right] w_{j},
\end{aligned}
$$

where the index $j$ refers to a cell center, and the operators $\Delta$ and $\nabla$ are forward and backward difference operators. The variable scaling factor $\lambda_{j+1 / 2}$ is defined as

$$
\lambda_{j+1 / 2}=\frac{1}{2}\left[\left(\bar{\lambda}_{\xi}\right)_{j}+\left(\bar{\lambda}_{\xi}\right)_{j+1}\right]
$$

where $\lambda_{\xi}$ is the largest eigenvalue in absolute value (i.e., spectral radius) of the flux Jacobian matrix associated with the the Euler equations. In two dimensions, with $(\xi, \eta)$ denoting arbitrary curvilinear coordinates, the scaling factor is usually defined as

$$
\left(\bar{\lambda}_{\xi}\right)_{j, k}=\phi_{j, k}(r)\left(\lambda_{\xi}\right)_{j, k}, \quad \phi_{j, k}(r)=1+r_{j, k}^{\zeta}
$$

with $r=\lambda_{\eta} / \lambda_{\xi}$. The exponent $\zeta$ is generally taken to be between $\frac{1}{2}$ and $\frac{2}{3}$. The coefficients $\varepsilon^{(2)}$ and $\varepsilon^{(4)}$ use the pressure as a sensor for sharp gradients, and they are defined as

$$
\begin{aligned}
\varepsilon_{j+1 / 2}^{(2)} & =\kappa^{(2)} \max \left(\nu_{j-1}, \nu_{j}, \nu_{j+1}, \nu_{j+2}\right), \\
\nu_{j} & =\left|\frac{p_{j-1}-2 p_{j}+p_{j+1}}{p_{j-1}+2 p_{j}+p_{j+1}}\right| \\
\varepsilon_{j+1 / 2}^{(4)} & =\max \left[0,\left(\kappa^{(4)}-\varepsilon_{j+1 / 2}^{(2)}\right)\right],
\end{aligned}
$$

where typical values for the constants $\kappa^{(2)}$ and $\kappa^{(4)}$ are in the ranges $\frac{1}{4}$ to $\frac{1}{2}$ and $\frac{1}{64}$ to $\frac{1}{32}$, respectively. We shall refer to Eq. (4) as the JST switch. The switching function $\nu$ can be interpreted as a limiter, in the sense that it maximizes the second-difference contribution at extrema and switches off the fourth-difference term. Moreover, at shock waves the dissipation is first order, and a first-order upwind scheme is produced for a scalar equation. In smooth regions of the flow field the dissipation is third order. 
Thus, we have two different dissipation mechanisms at work. The switch determines which one is active in any given region. For smooth flows, $\nu$ is small and the dissipation terms consist of a linear fourth difference that damps the high frequencies which the central difference scheme does not damp. This is necessary for achieving a steady state. In the neighborhood of large gradients in the pressure, $\nu$ becomes large and switches on the second-difference viscosity while simultaneously reducing the fourth-difference dissipation. This is needed to introduce an entropy condition so that the correct shock relations are satisfied and to reduce overshoots near discontinuities.

\section{Matrix Dissipation (MATD)}

Sharp resolution of shock waves without oscillations can be achieved by closely imitating an upwind scheme in the neighborhood of a shock wave. A key feature of upwind schemes is a matrix evaluation of the numerical dissipation. With this evaluation the dissipative terms of each discrete equation are scaled by the appropriate eigenvalues of the flux Jacobian matrix rather than by the spectral radius, as in the JST scheme. A matrix dissipation model can easily be constructed by starting with the JST formulation.

One can show [20] that the necessary modification of the JST scheme to produce a matrix dissipation model is the substitution of $|A|$ for the eigenvalue scaling factor $\lambda$ in Eqs. (2) and (3). Since the Euler equations are a strongly hyperbolic system, the coefficient matrix can be diagonalized. Assume $Q A Q^{-1}=\Lambda$ (diagonal matrix). Then $|A|$ is defined as $|A|=Q^{-1}|\Lambda| Q$ and $|\Lambda|=\operatorname{diag}\left(\left|\lambda_{1}\right|,\left|\lambda_{2}\right|,\left|\lambda_{3}\right|\right)$, where $\lambda_{i}$ are the forward acoustic, backward acoustic, and convective eigenvalues. Efficient ways of computing $|A|$ times a vector are presented in Ref. [20].

In practice one cannot choose $\lambda_{1}, \lambda_{2}, \lambda_{3}$ as the eigenvalues. Near stagnation points $\lambda_{3}$ approaches zero while near sonic lines $\lambda_{1}$ or $\lambda_{2}$ approaches zero. A zero artificial viscosity would create numerical difficulties. Hence, we limit these values as

$$
\begin{aligned}
\left|\tilde{\lambda}_{j}\right| & =\max \left(\left|\lambda_{j}\right|, V_{n} \rho(A)\right), \quad j=1,2 \\
\left|\tilde{\lambda}_{3}\right| & =\max \left(\left|\lambda_{3}\right|, V_{\ell} \rho(A)\right),
\end{aligned}
$$

where $\rho(A)$ is the spectral radius of $A$, and the linear eigenvalue $\lambda_{3}$ can be limited differently than the nonlinear eigenvalues. The parameters $V_{n}$ and $V_{\ell}$ have been determined by numerical experimentation. Typical values are $V_{n}=0.25$ and $V_{\ell}=0.025$.

\section{TVD and LED Properties}

In one dimension consider the approximation

$$
w_{j}^{n+1}=w_{j}-\frac{\Delta t}{2 \Delta x}\left(f_{j+1}-f_{j-1}\right)+\frac{\Delta t}{2 \Delta x}\left(Q_{j+1 / 2} \Delta w_{j+1 / 2}-Q_{j-1 / 2} \Delta w_{j-1 / 2}\right),
$$

where $\Delta w_{j+1 / 2}=w_{j+1}-w_{j}$. Suppose that the Jacobian matrix $A=\frac{\partial f}{\partial w}$ is at least locally constant. Then the scheme is TVD if

$$
Q_{j+1 / 2} \geq\left|A_{j+1 / 2}\right|
$$

This result follows directly from the fact that the system can be diagonalized, and we can ensure that each characteristic variable satisfies the TVD criterion (i.e., the total variation of the variable is nonincreasing). 
A replacement for the switch of Eq. (4) that is $T V D$ for a scalar equation is introduced in Ref. [20]. In one dimension this switch is given by

$$
\nu_{j}=\frac{\left|p_{j+1}-2 p_{j}+p_{j-1}\right|}{\left|p_{j+1}-p_{j}\right|+\left|p_{j}-p_{j-1}\right|+\epsilon} .
$$

and choose $\kappa^{(2)}=\frac{1}{2}$. In practice we usually use a weaker form than Eq. (6), for example,

$$
\nu_{j}=\frac{\left|p_{j+1}-2 p_{j}+p_{j-1}\right|}{(1-\omega) \mathcal{P}_{T V D}+\omega \mathcal{P}}
$$

where

$$
\begin{aligned}
\mathcal{P}_{T V D} & =\left|p_{j+1}-p_{j}\right|+\left|p_{j}-p_{j-1}\right| \\
\mathcal{P} & =p_{j+1}+2 p_{j}+p_{j-1},
\end{aligned}
$$

and $0 \leq \omega \leq 1$. The TVD switch of Eq. (6) is recovered when $\omega \ll 1$. Typically $\omega \sim 1 / 2$.

In Refs. [6] and [8] Jameson develops a theory for scalar nonoscillatory schemes based on the local extremum diminishing ( $L E D)$ principle that maxima do not increase and minima do not decrease. The LED principle applies to multidimensional problems, and it is equivalent to the TVD principle in one dimension. If a scheme is $L E D$, then the scheme is positive.

\section{SLIP Scheme}

Until now we have considered a combination of a low-order and high-order artificial viscosity based on a scalar switch. This switch has the disadvantage that it is based on only one quantity, the pressure. Moreover, it forces all variables to be treated equal, even though some experience sharp changes through the discontinuity while others are continuous across the shock. One can instcad limit independently each dependent variable in each coordinate direction.

Jameson [5] introduced a new class of limiters and implemented such a limiting process within a framework that he called the symmetric limited positive (SLIP) scheme. For the SLIP formulation Jameson constructed a family of limiter functions based on the function

$$
R(u, v)=1-\left|\frac{u-v}{|u|+|v|+\epsilon}\right|^{q}
$$

where $q$ is a positive number and $\epsilon$ has the dimensions of $u$. Note that $R(u, v) \approx 0$ whenever $u$ and $v$ have the opposite sign. Let $w$ be an element of the solution vector for the flow equations. According to our previous theory [20] $R\left(\Delta w_{j+3 / 2}, \Delta w_{j-1 / 2}\right)$, where $\Delta w_{j+3 / 2}=w_{j+2}-w_{j+1}$, would be replaced by $\nu_{j+1 / 2}$, where $\nu_{j+1 / 2}$ is the maximum of $\nu_{j}$ over the nearest neighbors and $\nu$ is given by Eq. (6). Define the limiter function $L(u, v)$ by

$$
L(u, v)=R(u, v) \frac{u+v}{2} .
$$

At the mesh cell interface $j+1 / 2$, we define the left and right states for each dependent variable as

$$
\begin{aligned}
& w_{L}=w_{j}+\frac{1}{2} L\left(\Delta w_{j+3 / 2}, \Delta w_{j-1 / 2}\right) \\
& w_{R}=w_{j+1}-\frac{1}{2} L\left(\Delta w_{j+3 / 2}, \Delta w_{j-1 / 2}\right)
\end{aligned}
$$


and so

$$
w_{R}-w_{L}=\Delta w_{j+1 / 2}-L\left(\Delta w_{j+3 / 2}, \Delta w_{j-1 / 2}\right)
$$

For the artificial viscosity all differences will be based on $w_{R}-w_{L}$. In the neighborhood of shock waves $R(u, v)$ and hence $L(u, v)$ are close to zero. Moreover, $w_{R}-w_{L}=\Delta w_{j+1 / 2}$, resulting in a first-order scheme for the artificial viscosity. For smooth flow $R(u, v) \simeq 1$, and $L(u, v)=(u+v) / 2$. Hence, in a smooth region

$$
\begin{aligned}
w_{R}-w_{L} & =\Delta w_{j+1 / 2}-L\left(\Delta w_{j+3 / 2}, \Delta w_{j-1 / 2}\right) \\
& \simeq \Delta w_{j+1 / 2}-\frac{\Delta w_{j+3 / 2}+\Delta w_{j-1 / 2}}{2} \\
& =-\frac{1}{2} \Delta^{3} w_{j+1 / 2} .
\end{aligned}
$$

Thus, the SLIP scheme has similar properties to the JST scheme. One can obtain the relationship between the SLIP and JST schemes by defining the diffusive flux $d_{j+1 / 2}$ as

$$
d_{j+1 / 2}=\alpha_{j+1 / 2}\left(w_{R}-w_{L}\right)
$$

with $\alpha_{j+1 / 2}=\kappa^{(2)} \lambda_{j+1 / 2}$. The quantity $\kappa^{(2)}$ is a parameter, and $\lambda$ is the spectral radius of the associated flux Jacobian matrix.

One difference between the JST and SLIP schemes involves the parameters $\kappa^{(2)}$ and $\kappa^{(4)}$ for the second and fourth differences, respectively. Both $\kappa^{(2)}$ and $\kappa^{(4)}$ are free parameters in the JST scheme. As seen from Eqs. (10) and (9) these parameters are automatically chosen as $\kappa^{(2)}$ and $\frac{1}{2} \kappa^{(2)}$ with the SLIP scheme. The coefficient of the second difference is chosen as $\frac{1}{2}$ so that the scheme is fully upwind for supersonic flows. However, the purpose of the fourthdifference viscosity is only to accelerate the convergence to a steady state by eliminating the odd-even point decoupling. Hence, we wish $\kappa^{(4)}$ to be as small as possible for accuracy while still achieving a good convergence rate. It does not seem reasonable to connect the two components of the artificial viscosity.

One can generalize the SLIP scheme by reintroducing a free parameter that essentially governs the level of the third-order viscosity in smooth regions. The resulting scheme has the disadvantage that a free parameter must be chosen; however, it has the advantages of greater flexibility and increased accuracy. We replace Eq. (7) by

$$
L(u, v, w)=R(u, w) \cdot\left[\left(1-4 \kappa^{(4)}\right) v+4 \kappa^{(4)} \frac{u+w}{2}\right]
$$

where left and right state values are determined by

$$
\begin{aligned}
& w_{L}=w_{j}+\frac{1}{2} L\left(\Delta w_{j+3 / 2}, \Delta w_{j+1 / 2}, \Delta w_{j-1 / 2}\right) \\
& w_{R}=w_{j+1}-\frac{1}{2} L\left(\Delta w_{j+3 / 2}, \Delta w_{j+1 / 2}, \Delta w_{j-1 / 2}\right) .
\end{aligned}
$$

When $\kappa^{(4)}=\frac{1}{4}$, the $L$ of Eq. (11) reduces to the original $L$ of Eq. (7). Now, at shock waves $R(u, w)$ is small and we have $w_{R}-w_{L}=\Delta w_{j+1 / 2}$. For smooth regions of the flow field we have $w_{R}-w_{L}=-2 \kappa^{(4)} \Delta^{3} w_{j+1 / 2}$. 


\section{CUSP/AUSM Scheme}

So far we have described the use of an artificial viscosity based on either a scalar or matrix coefficient. Liou and coworkers designed a scheme called Advection Upstream Splitting Method (AUSM) $[10,11,12]$. This method was later refined for large-scale 3-D viscous computations [15]. AUSM is based on a splitting of the flux function into convective and pressure contributions. In some sense, the pressure terms contribute to the acoustic waves while the velocity terms contribute to convective waves. Hence, it is reasonable that these flux terms be treated differently. Liou thus considers decompositions of the flux vector that are not based on a characteristic decomposition but on Mach number scaled contributions of the left and the right states to the interface flux. This decomposition has the disadvantage that it is more difficult to develop for other sets of equations compared with a characteristic decomposition. A similar type scheme called the convective upwind split pressure (CUSP) scheme was later introduced by Jameson [5] and subsequently modified by Tatsumi, Martinelli, and Jameson [7, 8, 22, 23]. The CUSP scheme has several advantages. First, one can consider the scheme as another type of artificial viscosity, since it is defined as a sum of the central flux average plus a dissipative flux. Hence, it can be readily used with a variety of time-stepping schemes (e.g., multistage, $L U$, implicit, etc.). Second, the CUSP formulation can be used with multistage schemes which do not evaluate the artificial dissipation fluxes at every stage, in order to reduce computational work. Another advantage of the CUSP scheme is that it can be easily combined with preconditioning, since preconditioning is based on the inviscid flux form and not the artificial dissipation. Hence, we shall only describe the CUSP version of this type of scheme.

\section{Definition of CUSP Scheme}

Previously, we introduced the scalar and matrix-valued viscosities by considering $d_{j+1 / 2}$ of the form

$$
d_{j+1 / 2}=\frac{1}{2} Q_{j+1 / 2}\left(w_{j+1}-w_{j}\right) .
$$

The factor $\frac{1}{2}$ is introduced so that we get first-order upwinding when $Q_{j+1 / 2}=I$. We note that for the scheme to be $T V D, Q$ must be sufficiently large (see Eq. (5)). For the matrix viscosity we chose $Q=|A|$ (modified near zero eigenvalues) while for the scalar viscosity we chose $Q=\sigma(A) I$.

For the CUSP scheme we instead choose $d$ as a linear combination of $w$ and $f$. We shall only consider the choice for the state vector given by

$$
\begin{aligned}
w_{h} & =(\rho \rho u \rho H)^{T} \\
f & =u\left(\begin{array}{c}
\rho \\
\rho u \\
\rho H
\end{array}\right)+\left(\begin{array}{l}
0 \\
p \\
0
\end{array}\right)=u w_{h}+f_{p} .
\end{aligned}
$$

This choice is denoted HCUSP by Jameson [7]. The first-order accurate CUSP scheme is defined as

$$
d_{j+1 / 2}=\frac{1}{2} \nu c\left(w_{j+1}-w_{j}\right)+\frac{\beta}{2}\left(f_{j+1}-f_{j}\right)
$$

The factor $c$ is included so that $\nu$ is dimensionless. We thus consider only scalar parameters instead of a matrix coefficient, but we have two free parameters, $\nu$ and $\beta$. The scheme is total 
enthalpy preserving. By using the arithmetic average, $\bar{u}=\frac{1}{2}\left(u_{j+1}+u_{j}\right)$, and the definition

$$
\alpha c=\nu c+\beta \bar{u},
$$

one can rearrange Eq. (12) to obtain

$$
d_{j+1 / 2}=\frac{1}{2} \alpha c\left(w_{j+1}-w_{j}\right)+\frac{\beta}{2}\left(f_{p_{j+1}}-f_{p_{j}}\right)+\frac{\beta}{2} \bar{w}\left(u_{j+1}-u_{j}\right) .
$$

Introducing the Roe matrix [17] $A_{R L}$, we have that $f_{R}-f_{L}=A_{R L}\left(w_{R}-w_{L}\right)$. This relation is exact if $A_{R L}$ is computed from weighted averages of the left and the right states. Then the first-order dissipation is

$$
d_{j+1 / 2}=\frac{1}{2}\left(\beta A_{R L}+\nu c I\right)\left(w_{R}-w_{L}\right) .
$$

We see from this formula that $d$ is a linear function of $A$. Recall that $|A|$ is a quadratic function of $A$, by the Cayley-Hamilton Theorem. Hence, it is not possible to bound $d$ by $|A|$. Thus, the CUSP scheme cannot be TVD.

Assume that the subscript $L$ denotes the interior point inside the shock zone, $R$ is the state downstream of the shock, and the state $L R$ is subsonic. Jameson [7] shows that the downstream point with the state $R$ is in equilibrium if

$$
f_{R}-f_{L}+\frac{\nu c}{1+\beta}\left(w_{R}-w_{L}\right)=0
$$

Substituting the Roe matrix for the difference in $f$ into Eq. (15) we get

$$
\left(A_{R L}+\frac{\nu c}{1+\beta} I\right)\left(w_{R}-w_{L}\right)=0
$$

Hence, $w_{R}-w_{L}$ is an eigenvector of $A_{R L}$, and $-(\nu c) /(1+\beta)$ is the corresponding eigenvalue. The eigenvalues of $A_{R L}$ are known to be $\lambda^{+}, \lambda^{-}$and $u$. If $\lambda$ is an eigenvalue of $A$, then using this formula for $\nu c$ in Eq. (14) we have

$$
d_{j+1 / 2}=\frac{1}{2}\left[-\lambda I+\beta\left(A_{R L}-\lambda I\right)\right]\left(w_{R}-w_{L}\right)
$$

In order to have a positive diffusion when $u>0$, we require that $\lambda$ be negative (i.e., $-(\nu c) /(1+$ $\beta)=\lambda^{-}$). Thus,

$$
\nu c=-(1+\beta) \lambda^{-}
$$

For $u<0$ we obtain similarly

$$
\nu c=(1-\beta) \lambda^{+}
$$

So, we have reduced our two free parameters to one free parameter by demanding a one point shock profile. More generally, Jameson shows that one obtains a shock profile with one interior point if the following two conditions hold: 
I. When the flow is supersonic through the shock then one obtains a totally upwind flux.

II. The artificial dissipation $Q$ satisfies a generalized eigenvalue problem

$$
\left(A_{R L}-\alpha_{R A} Q_{R A}\right)\left(w_{R}-w_{A}\right)=0
$$

at the exit from the shock.

The second condition is satisfied by both the matrix viscosity and the CUSP scheme; however, the scalar viscosity does not satisfy the first condition. We again note that the TVD condition $Q \geq|A|$ is satisfied by the scalar and matrix viscosities but not by CUSP.

What remains to be done is to choose suitable functions for $\beta$ and $\nu c$ which satisfy the above requirements. Jameson's choice for $\beta$, which is based upon the eigenvalues corresponding to the acoustic waves, is given by

$$
\beta=\left\{\begin{array}{ccc}
+\max \left(0, \frac{u+\lambda^{-}}{u-\lambda^{-}}\right) & \text {if } & 0 \leq M<1 \\
-\max \left(0, \frac{u+\lambda^{+}}{u-\lambda^{+}}\right) & \text {if } & -1<M<0 \\
\operatorname{sgn}(M) & \text { if } & |M| \geq 1 .
\end{array}\right.
$$

The cutoffs, $\beta \geq 0$ for $u>0$ and $\beta \leq 0$ for $u<0$, ensure that the pressure terms are discretized centrally for small Mach numbers. Shock capturing with one interior point is obtained by taking

$$
\nu c=\left\{\begin{array}{ccc}
|u| & \text { if } & \beta=0 \\
-(1+\beta) \lambda^{-} & \text {if } & \beta>0,0<M<1 \\
(1-\beta) \lambda^{+} & \text {if } & \beta<0,-1<M<0 \\
0 & \text { if } & |M| \geq 1 .
\end{array}\right.
$$

The dissipation coefficients are to be computed from Roe-averaged quantities. They provide full upwinding for supersonic flow $(\beta=\operatorname{sgn}(M), \nu=0)$. The choice of $\nu c=|u|$ for $\beta=0$ yields a continuous dissipation coefficient in the subsonic region, and it does not smear slip lines with $|u|$ close to zero. This makes the CUSP formulation attractive for viscous flow calculations with boundary layers. However, viscous flows are usually discretized by using cells with large aspect ratio. It is well known that this situation requires larger dissipation scaling in the direction of the long cell sides than given by $|u|$. We redefine the dissipation coefficients in the individual coordinate directions. For the $\boldsymbol{\xi}$-direction we have

$$
\nu c_{\xi}=r^{+}\left\{\begin{array}{ccc}
\max \left(|u|, \delta c r^{-}\right) & \text {if } & \beta=0 \\
-(1+\beta) \lambda^{-} & \text {if } & \beta>0 \text { and } \\
& & 0<M<1 \\
(1-\beta) \lambda^{+} & \text {if } & \beta<0 \text { and } \\
& & -1<M<0 \\
0 & \text { if } & |M| \geq 1 .
\end{array}\right.
$$

where $r^{+}$and $r^{-}$are functions of the spectral radii in the $\xi$ and $\eta$ directions $\left(\lambda_{\xi}\right.$ and $\left.\lambda_{\eta}\right)$, and they are defined as follows:

$$
r=\left(\frac{\lambda_{\eta}}{\lambda_{\xi}}\right)^{\zeta}, \quad r^{+}=\max (r, 1), \quad r^{-}=\min (r, 1)
$$

The dissipation coefficient in the $\eta$-direction is defined correspondingly. 


\section{Simplified Scheme}

Several modifications of the CUSP scheme have been in use so far. Based upon the $w_{h}=$ $(\rho \rho u \rho H)^{T}$ system the dissipation coefficients presented in Refs. [7] and [23] are as follows:

$$
\begin{aligned}
& \alpha=\left\{\begin{array}{cll}
|M| & \text { if } & |M| \geq \epsilon \\
\frac{1}{2}\left(\epsilon+\frac{M^{2}}{\epsilon}\right) & \text { if } & |M|<\epsilon,
\end{array}\right. \\
& \beta=\left\{\begin{array}{ccc}
+\max \left(0, \frac{u+\lambda^{-}}{u-\lambda^{-}}\right) & \text {if } & 0 \leq M<1 \\
-\max \left(0, \frac{u+\lambda^{+}}{u-\lambda^{+}}\right) & \text {if } & -1<M<0 \\
\operatorname{sgn}(M) & \text { if } & |M| \geq 1 .
\end{array}\right.
\end{aligned}
$$

This choice does not allow exact shock capturing because Eqs. (17) and (18) are not satisfied. Furthermore, Roe averaging has been replaced by arithmetic averaging in Ref. [7] and $\lambda^{-}, \lambda^{+}$ by $u-c, u+c$, respectively. This simplification saves a few square roots in the coding of the dissipative flux. This then becomes

$$
\begin{aligned}
& \alpha=\left\{\begin{array}{cll}
|M| & \text { if } & |M| \geq \epsilon \\
\frac{1}{2}\left(\epsilon+\frac{M^{2}}{\epsilon}\right) & \text { if } & |M|<\epsilon,
\end{array}\right. \\
& \beta=\left\{\begin{array}{ccc}
\max (0,2 M-1) & \text { if } & 0 \leq M<1 \\
\min (0,2 M+1) & \text { if } & -1<M<0 \\
\operatorname{sgn}(M) & \text { if } & |M| \geq 1 .
\end{array}\right.
\end{aligned}
$$

\section{Higher Order Scheme}

Having determined $\nu c$ and $\beta$, we see from Eq. (12) that the scheme is completely defined in terms of $w$ and $f$. Recalling from previous sections, we now need to combine a first-order artificial dissipation with a high-order dissipation. As previously the high-order dissipation is used in the smooth regions while the low-order artificial viscosity is used in the neighborhood of shocks. To allow changing from one type to the other we can either use the JST switch of Eq. (4) or the SLIP scheme. Formula (12), as given, is only first-order accurate, as it depends only on $d_{j+1 / 2}=w_{j+1}-w_{j}$, and so the complete artificial viscosity behaves like a second difference. In order to improve this situation we need to use a MUSCL or SLIP approach to get the first difference to higher order accuracy. Since the fluxes in the CUSP scheme have different slopes on the two sides of the sonic line, the SLIP construction, which relies on successive differences, can cause a loss of smoothness at the sonic line. This difficulty can be avoided by using the MUSCL approach to obtain the states corresponding to higher order accuracy. To impose monotonicity we can apply the limiter of the SLIP scheme. In particular, we can replace Eq. (12) by

$$
d_{j+1 / 2}=\frac{\nu c}{2}\left(w_{R}-w_{L}\right)+\frac{\beta}{2}\left[f\left(w_{R}\right)-f\left(w_{L}\right)\right],
$$

where $w_{R}, w_{L}$ are given by Eq. (8). This procedure was followed throughout the numerical examples shown below. Application of Eq. (8) to the $w_{h}=(\rho \rho u \rho H)^{T}$ variables still allows total enthalpy to be preserved in the higher order scheme. 


\section{Analysis of CUSP Scheme}

The eigenvalues of $\beta A_{R L}+\nu c I$ in Eq. (14) are $\mu_{1} c, \mu_{2} c$, and $\mu_{3} c$. Using the simplifications of Eq. (22) the eigenvalues are:

$$
\begin{aligned}
& \mu_{1}=|M|, \\
& \mu_{2}=\left\{\begin{array}{ccc}
|M| & \text { if } & |M|<\frac{1}{2} \\
\alpha+\beta & \text { if } & \frac{1}{2} \leq M \leq 1 \\
|M+1| & \text { if } & |M|>1,
\end{array}\right. \\
& \mu_{3}=\left\{\begin{array}{ccc}
|M| & \text { if } & |M|<\frac{1}{2} \\
\alpha-\beta & \text { if } & \frac{1}{2} \leq M \leq 1 \\
|M-1| & \text { if } & |M|>1
\end{array}\right.
\end{aligned}
$$

We note that for $|M|<\frac{1}{2}$ all three eigenvalues of the artificial viscosity are equal, and so we have the equivalent of a scalar viscosity. The scalar viscosity now scales with $|M| c$ rather than $(|M|+1) c$ for the JST scalar viscosity. This is more similar to the case of preconditioning where all the eigenvalues are approximately $|M| c$ for low speed flow. Hence, we expect that the CUSP dissipation should work properly for very low Mach numbers provided the central flux terms are augmented by a suitable preconditioning matrix.

In the subsonic range where $\beta=0$, all of the versions of the CUSP scheme do not satisfy Eqs. (17) and (18), which are necessary for shock capturing. That is, the cell-face Mach numbers in the shock structure have to be larger than about 0.5 in order to avoid post-shock oscillations. The motivation to design $\nu c=|u|$ for $\beta=0$ has already been discussed previously and is not repeated here. However, the choice of the function for $\beta$, as given in Eq. (19), is not necessarily optimal. For example, $\beta=\max \left(0,\left(u+\frac{1}{2} \lambda^{-}\right) /\left(u-\lambda^{-}\right)\right)$would allow shock capturing for Mach numbers down to about $1 / 3$, but the subsonic dissipation would be twice as large, $\nu c=2|u|$ for $\beta=0$. Nevertheless, our own experience gained from a number of numerical applications suggests that there is no need for further modifications of $\beta$.

It is rather difficult to compare the effect of the parameter $\kappa^{(4)}$ of the JST and the combined CUSP/SLIP schemes since these schemes also include eigenvalue information which is not the same. To isolate the effect we consider a low Mach number flow with preconditioning (see Ref. [25] for details). Now both switches are based on the convective eigenvalue $u$. A typical value for the $J S T$ scheme is $\kappa^{(4)}=\frac{1}{32}$. However, for a aspect ratio of one the Martinelli scaling [13] adds another factor of two. The parameter $\alpha=0$ in the preconditioning adds an additional factor of approximately 2.6. Hence, the effective constant multiplying the fourth difference is about $\frac{5}{32}$, which is somewhat smaller than the $\frac{1}{4}$ used with the original CUSP scheme with the SLIP formulation. For transonic flows it is more difficult to compare the levels of dissipation. However, it seems that the original SLIP scheme yields too high a viscosity level and so $\kappa^{(4)}$ should be reduced to less than $\frac{1}{4}$. Numerical computations demonstrate the improved accuracy (though slower convergence) for standard transonic turbulent flows when $\kappa^{(4)}$ is reduced.

\section{Numerical Results}

In this section we assess the accuracy and shock capturing capabilities of the HCUSP scheme. Comparisons are made between the HCUSP and MATD schemes. The commonly used scalar dissipation scheme is also included in some of the comparisons. In so doing one can clearly see 

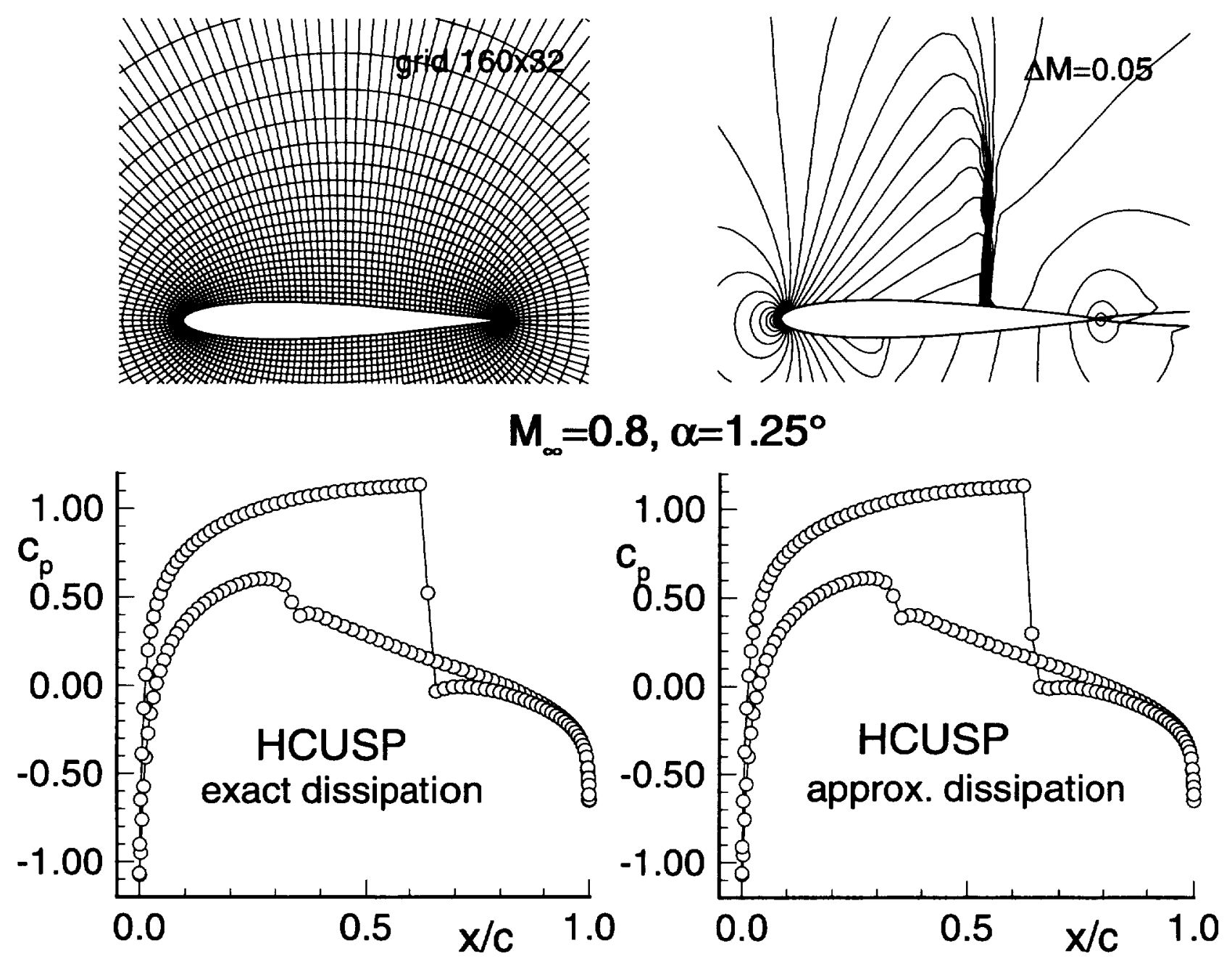

Figure 1: Influence of HCUSP dissipation coefficients on transonic flow over NACA 0012 airfoil. 


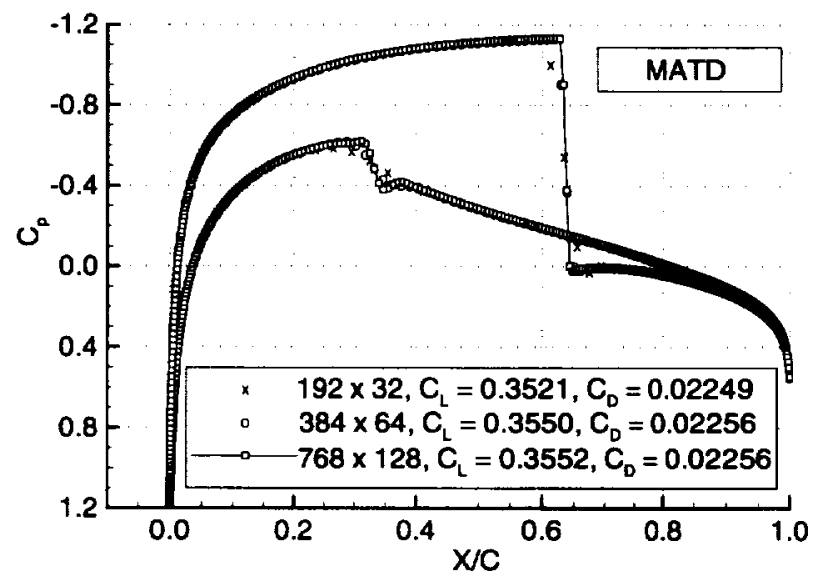

Figure 2a: Inviscid pressure distributions computed with MATD scheme (NACA 0012 airfoil, $M=0.80, \alpha=1.25^{\circ}$ ).

the superiority of the high-resolution HCUSP and MATD schemes on even relatively coarse meshes (i.e., 16 cells in the boundary layer of a viscous flow). The flow problems considered in the evaluation of these numerical diffusion schemes include the following: 1) Inviscid flow over airfoils, 2) laminar flow over a flat plate, 3) turbulent flow over an airfoil, 4) inviscid and viscous hypersonic flow over a 2 -D wedge. The computational effort and convergence behavior in computing these solutions is given. In all cases a five-stage Runge-Kutta scheme in conjunction with the convergence acceleration techniques of local time stepping, implicit residual smoothing, and multigrid was used.

The first case is similar to the application published in Ref. [7]. Results for inviscid transonic flow over the NACA 0012 airfoil are shown in Fig. 1. The free-stream Mach number is 0.8 and the angle of attack is $1.25^{\circ}$. An O-topology mesh of $160 \times 32$ cells was used and is partially depicted in Fig. 1. The shock waves on the upper and lower surfaces are captured with a single interior point. The differences between the accurate dissipation coefficients of Eqs. (19) and (20) on the one hand and the simplified coefficients of Eq. (22) on the other are small. Careful examination reveals that the exact formulation is somewhat more accurate on the upper surface where the shock is stronger. These inviscid solutions were obtained with a 4-level multigrid and sawtooth-type cycle. They converged at a rate of about 0.90 per multigrid cycle.

In Figs. 2 and 3 the HCUSP and MATD schemes are compared for this case. Solutions were computed on three successively finer C-topology meshes. The coarsest mesh contained $192 \times 32$ cells, with 160 cells on the airfoil, and for each sequential mesh the number of cells in each coordinate direction was doubled. The principal differences between the solutions occur at the shock waves. Since the MATD scheme uses a pressure switch for all the flow equations, it cannot capture a shock with a single interior point. It requires three interior points. Nevertheless, the resolution of the stronger upper surface shock is nearly the same for both the MATD and HCUSP schemes on the $384 \times 64$ and $768 \times 128$ meshes. With the MATD formulation there is some smearing on the $192 \times 32$ mesh. As clearly evident in Fig. 3 the HCUSP scheme allows a sharp definition of the weak lower surface shock and the Zierep singularity that immediately follows. The aerodynamic coefficients calculated with the MATD scheme on the $384 \times 64$ mesh essentially agree with those for the finest grid. The lift and drag coefficients determined with the HCUSP scheme on the corresponding meshes are not the 


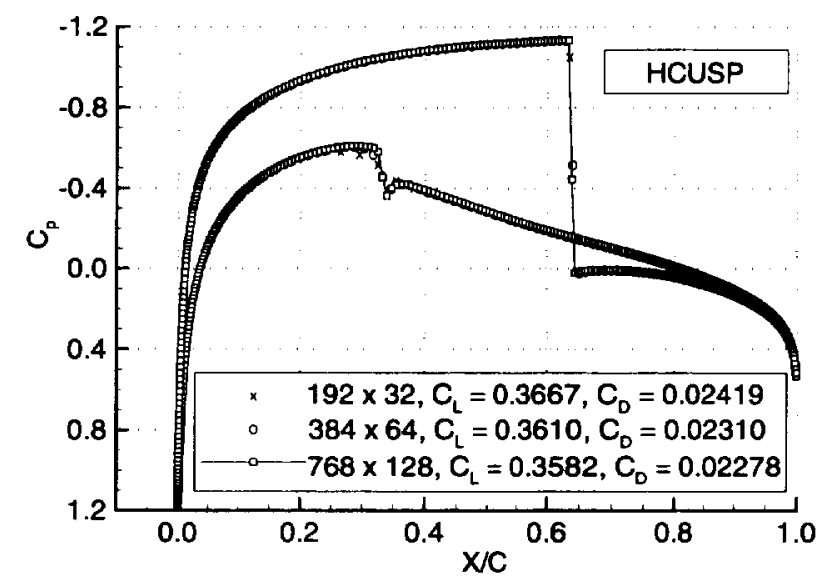

Figure 2b: Inviscid pressure distributions computed with HCUSP scheme (NACA 0012 airfoil, $M=0.80, \alpha=1.25^{\circ}$ ).

same, with the finest grid values approaching those obtained with the MATD scheme. These results probably reflect the higher level of background dissipation with the HCUSP scheme.

As an initial evaluation of the dissipation schemes for viscous flows we consider low-speed $\left(M_{\infty}=0.15\right)$ flow over a flat plate at zero incidence. For this flow the Reynolds number per unit length is $10^{5}$. The computational domain is a rectangle. With respect to the leading edge of the plate, the domain extends two plate lengths upstream and one plate length downstream. The upper boundary is four plate lengths above the plate. Solutions were computed on the same domain and grids used in Ref. [23]. Starting with the finest mesh, coarser meshes were determined by successively eliminating every other mesh line. The finest grid consists of $512 \times 128$ cells, with 384 cells on the plate. In the direction $y$ normal to the plate the grid is spaced uniformly in the boundary-layer coordinate $\eta\left(\eta=y / R e_{x}^{1 / 2}\right)$, where $x$ is the coordinate parallel to the surface, and $R e_{x}$ is the Reynolds number based on distance from the leading edge of the plate). Thus, there is constant resolution of the boundary layer at each location along the plate. Outside the boundary layer the grid is stretched exponentially. In order to resolve the region in the vicinity of the stagnation point, the grid is clustered at the leading edge of the plate. At the surface of the plate no-slip and adiabatic boundary conditions are enforced. Along the boundary upstream of the leading edge, a symmetry condition is applied. Characteristic type boundary conditions are used at the upstream, downstream, and upper boundaries.

A comparison of the velocity profile at $X / L=0.82$ computed with the the scalar, matrix, and HCUSP dissipation forms is displayed in Fig. 4. Even with just eight points in the boundary layer (64 × 16 grid) the MATD and HCUSP schemes nearly replicate the Blasius solution. As demonstrated in Ref. [1] scalar dissipation can produce serious contamination. With the scalar dissipation, more than 32 points are required in the boundary layer to obtain a grid converged solution. For the MATD and HCUSP schemes the variation of the errors (relative to the Blasius solution) in the calculated skin friction, displacement thickness, and momentum thickness are shown in Figs. 5a and 5b. The standard definitions [18] are used for these boundary-layer quantities. The errors in all the boundary-layer parameters are quite similar for the highresolution schemes. This is not surprising since both schemes have a scaling factor that vanishes 


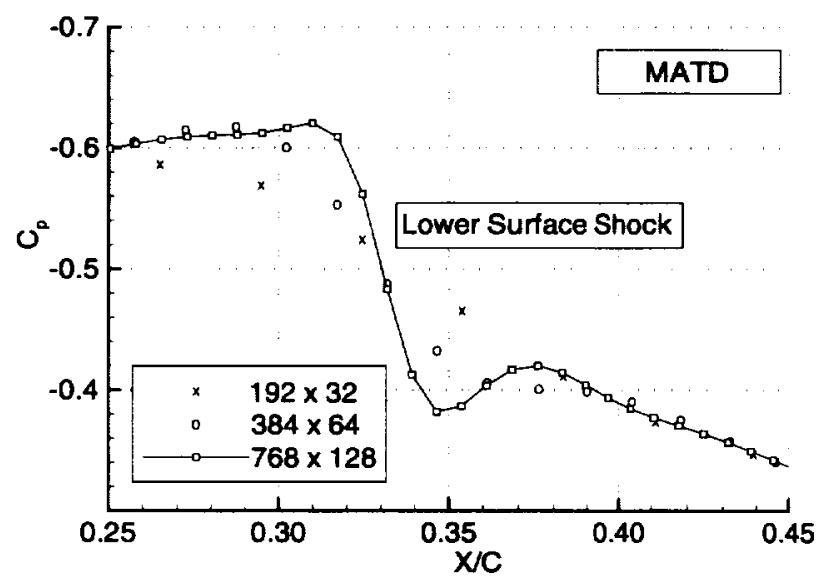

Figure 3a: MATD solutions near lower surface shock (NACA 0012 airfoil, $M=0.80, \alpha=$ $\left.1.25^{\circ}\right)$.

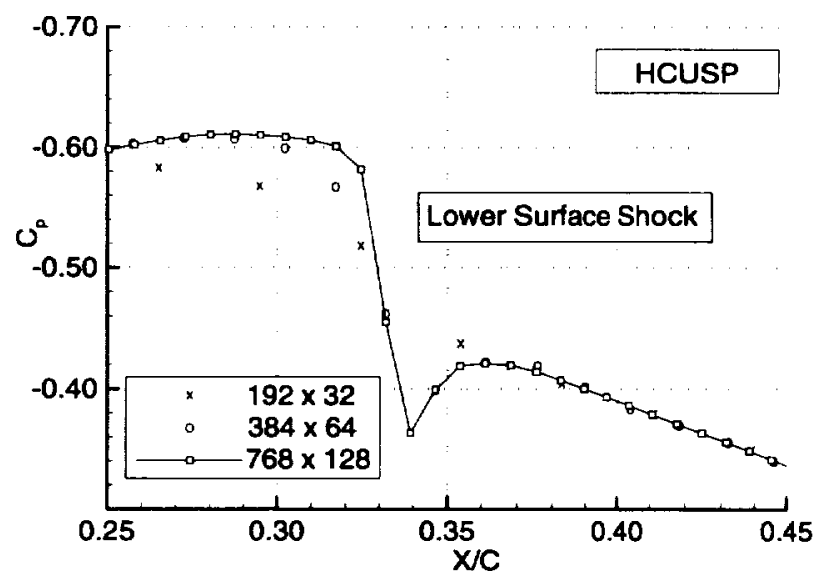

Figure 3b: HCUSP solutions near lower surface shock (NACA 0012 airfoil, $M=0.80, \alpha=$ $\left.1.25^{\circ}\right)$. 

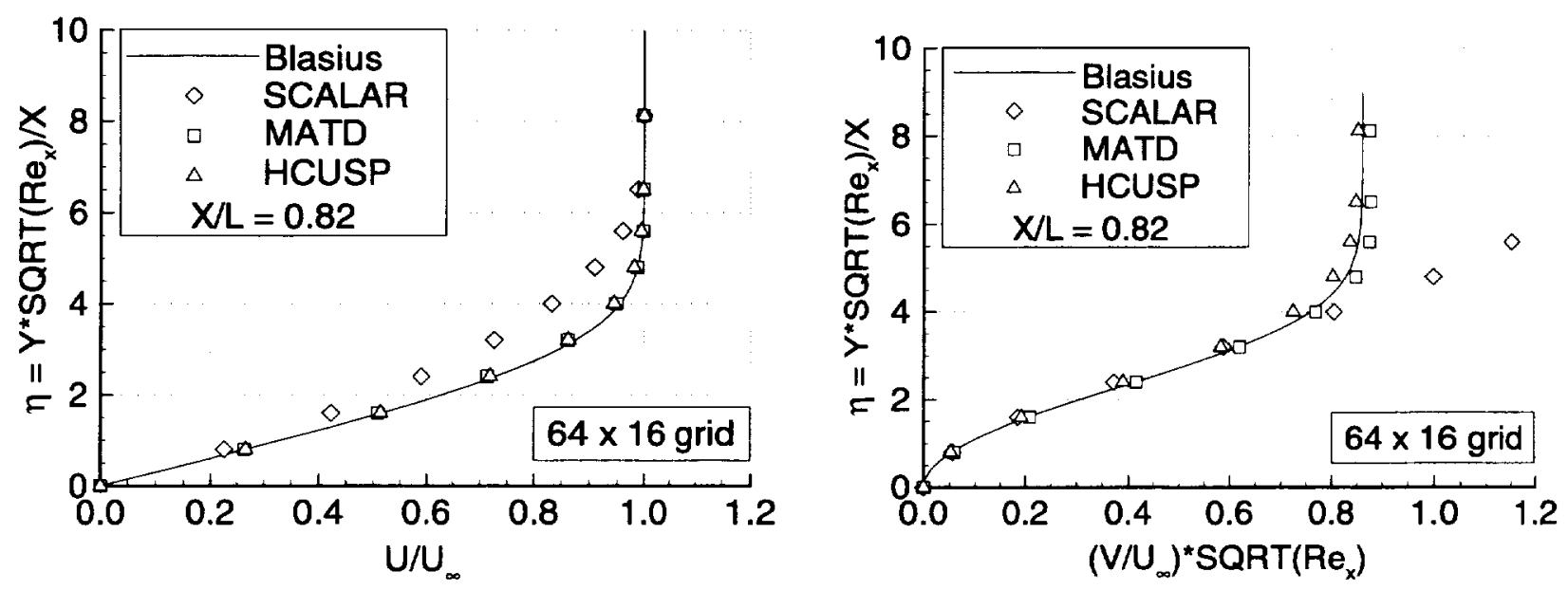

4a: Tangential and transverse velocity profiles, $\mathrm{X} / \mathrm{L}=0.82,64 \times 16$ grid.
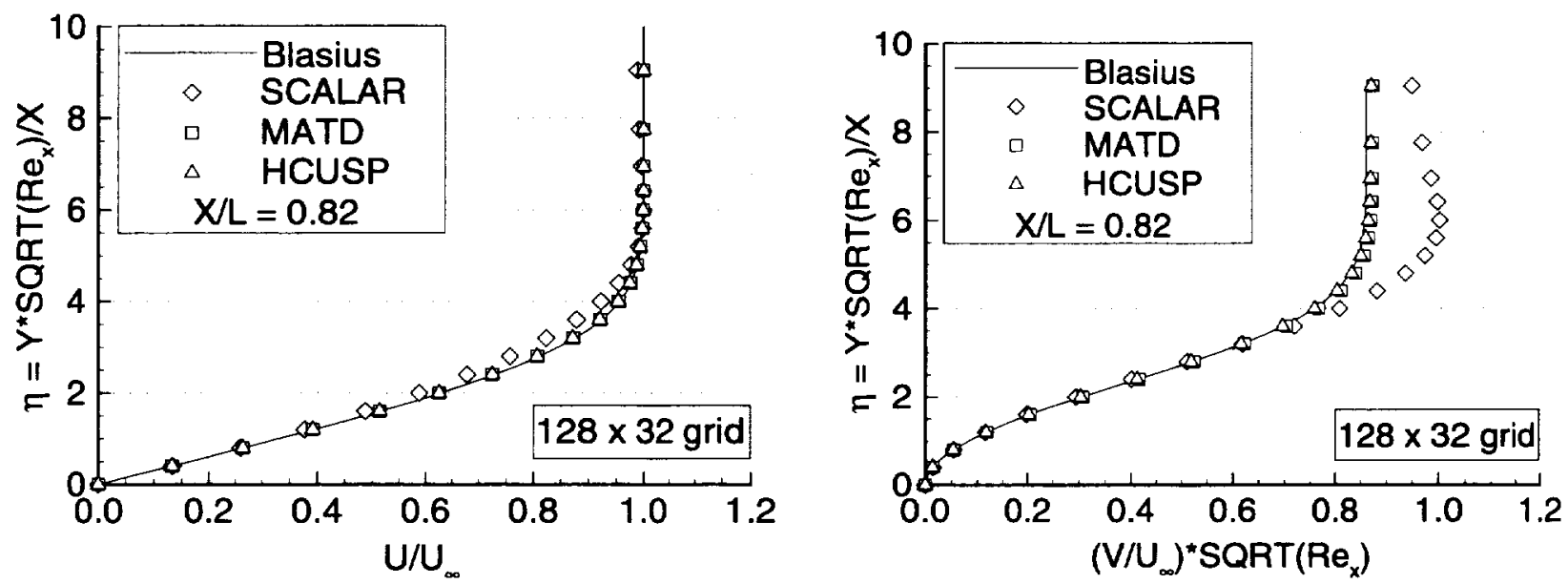

4b: Tangential and transverse velocity profiles, $\mathrm{X} / \mathrm{L}=0.82,128 \times 32$ grid.
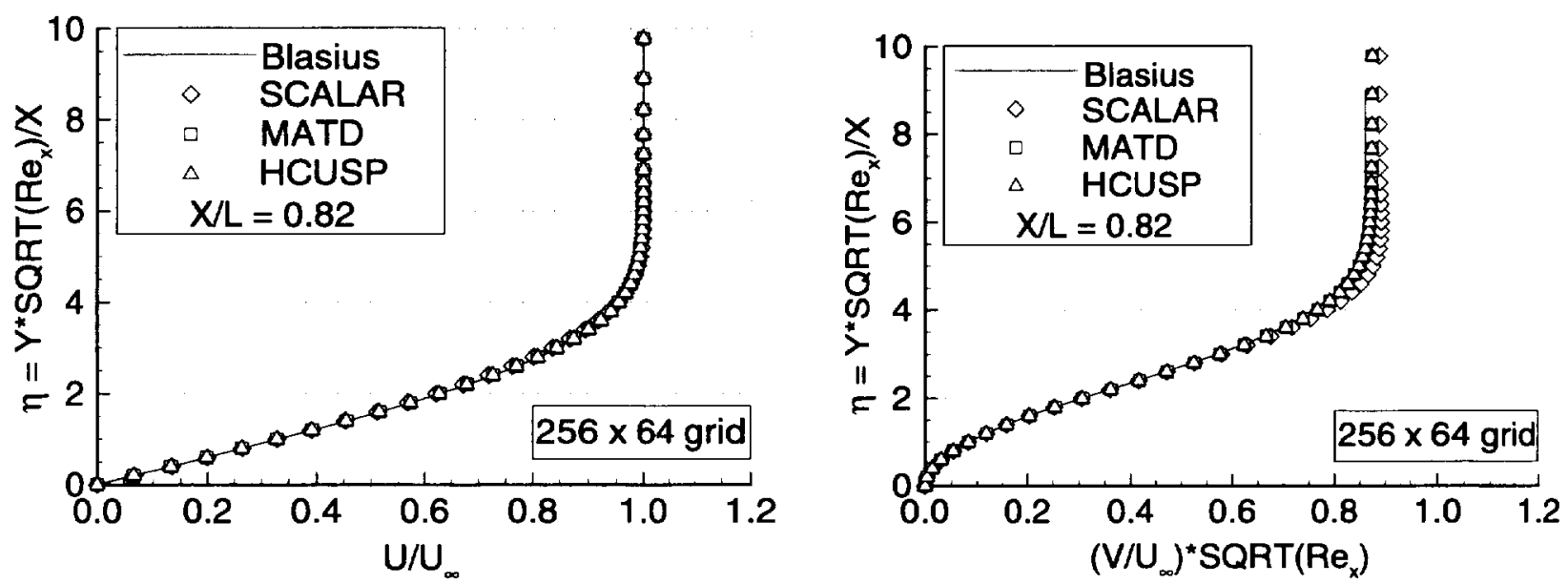

4c: Tangential and transverse velocity profiles, $\mathrm{X} / \mathrm{L}=0.82,256 \times 64$ grid.

Figure 4: Boundary-layer profiles on flat plate with $M=0.15$ and $R e=10^{5}$. 


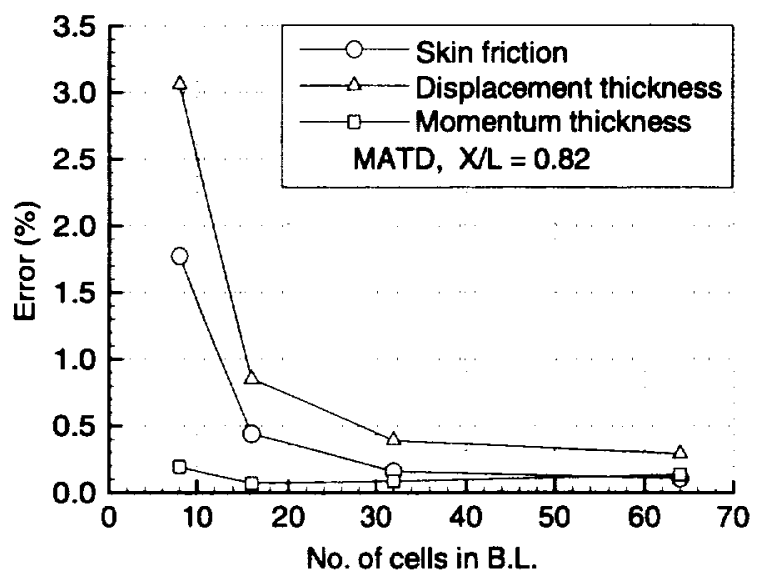

Figure 5a: Comparison of MATD scheme results with Blasius solution $(M=0.15$ and $R e=$ $\left.10^{5}\right)$.

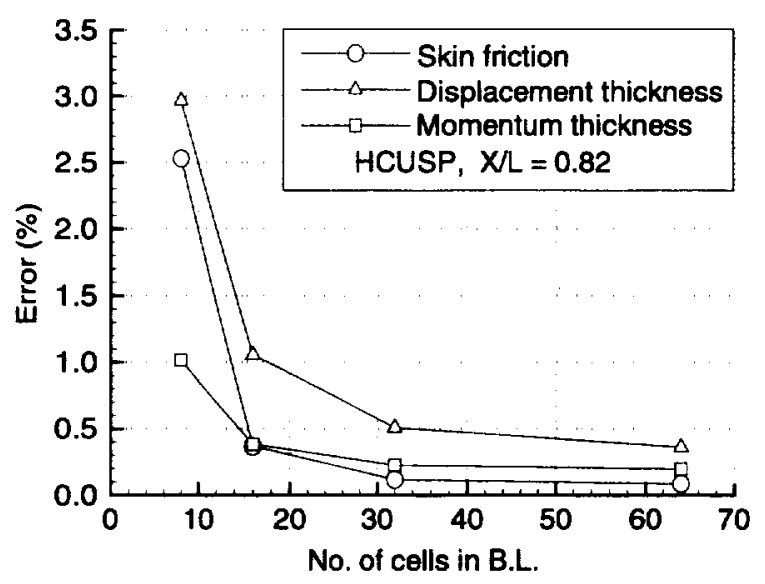

Figure 5b: Comparison of HCUSP scheme results with Blasius solution $(M=0.15$ and $R e=$ $\left.10^{5}\right)$. 


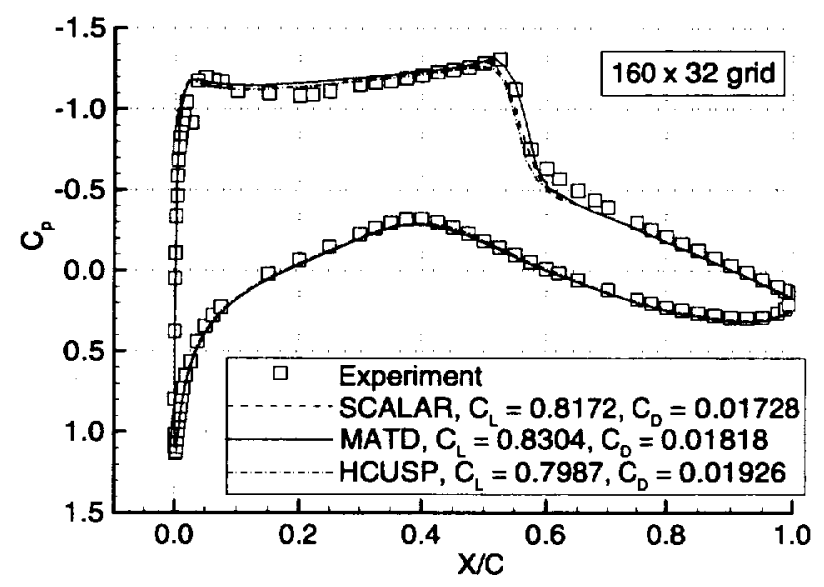

Figure 6a: Comparison of pressure distributions with SCALAR, MATD, and HCUSP schemes on $160 \times 32$ grid (RAE 2822 airfoil, $M=0.73, \alpha=2.79^{\circ}, R e=6.5 \times 10^{6}$ ).

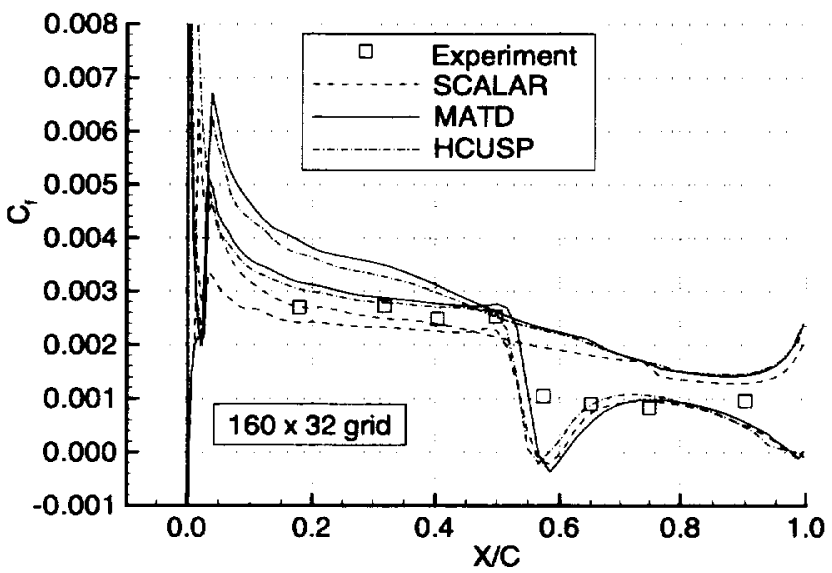

Figure 6b: Comparison of skin-friction distributions with SCALAR, MATD, and HCUSP schemes on $160 \times 32$ grid (RAE 2822 airfoil, $M=0.73, \alpha=2.79^{\circ}, R e=6.5 \times 10^{6}$ ). 
as the surface is approached.

We next consider transonic flow over the RAE 2822 airfoil. The free-stream Mach number is 0.73 , the angle of attack is $2.79^{\circ}$, and the Reynolds number based on the airfoil chord is $6.5 \times 10^{6}$. Transition from laminar to turbulent flow is fixed at the $3 \%$ chord location. The C-type grids used are as follows: (1) $160 \times 32$ with 128 cells on the airfoil, (2) $320 \times 64$ with 256 cells on the airfoil, and (3) $640 \times 128$ with 512 cells on the airfoil. The outer boundary is located 20 chords from the airfoil. The normal spacing at the surface of the $640 \times 128$ mesh is $7.5 \times 10^{-6}$ chords. At the leading and trailing edges of the airfoil the mesh is clustered, giving tangential spacings of $1.17 \times 10^{-3}$ and $1.86 \times 10^{-3}$ chords, respectively. These spacings are roughly doubled with each mesh coarsening. To determine the effect of further mesh refinement a calculation was performed with the MATD scheme on a $1280 \times 256$ grid.

In Figs. 6-8 the pressure $\left(C_{p}\right)$ and surface skin-friction $\left(C_{f}\right)$ distributions computed with the different dissipation schemes for the range of meshes described are shown, along with the experimental data [2]. As in the inviscid cases the primary differences in the solutions occur at the shock wave. On the coarsest grid $(160 \times 32)$ both the SCALAR and HCUSP schemes produce a solution with the shock too far upstream. This is an unexpected result for the HCUSP scheme. The acceleration of the flow upstream of the shock is underpredicted relative to the finest grid. In Ref. [21] the adverse effect of a smooth limiter on the accuracy of the solution in the vicinity of flow transition, and thus on the acceleration of the flow upstream of the shock, is demonstrated. Therefore, such a result with the HCUSP scheme could be a consequence of the smooth limiter being used. With both the SCALAR and the MATD schemes a nonphysical increase in the skin-friction solution on the upper surface appears at the trailing edge of the airfoil. This behavior does not occur in the solution obtained with the HCUSP scheme. The computed aerodynamic coefficients, including the pressure and friction contributions to the total drag, are given in Table 1. On each mesh the lift and drag coefficients corresponding to the solution obtained with the MATD scheme exhibit the closest agreement with the $1280 \times 256$ grid values. There are only small discrepancies in the coefficients associated with the MATD and HCUSP schemes on the $640 \times 128$ grid.

Convergence behavior for the HCUSP and MATD schemes is displayed in Fig. 9. Five levels of multigrid were used for this case and either 50 or 70 cycles werc executed on two coarser meshes in order to obtain an initial solution. On the $320 \times 64$ grid the average rate of reduction of the residual for both schemes is about 0.92 . Figure 9 also shows the effect of the modification given by Eq. (20) to $\nu c$ in the HCUSP scheme. The convergence is improved by using the 2-D formulation for the dissipation coefficient $\nu c$. Note that convergence with $\zeta=0$ was possible for this transonic case but not for the hypersonic case presented below.

The fourth case is hypersonic 2-D flow over a blunt wedge. Figure 10 displays the solutions obtained for viscous and inviscid flow using identical meshes of $64 \times 48$ cells. Physical diffusion is so large that the shock profile is significantly smeared in the viscous result. For inviscid flow, on the other hand, we obtain perfect capturing with a single interior point in the shock structure by using the formulation of Eqs. (19) and (20). Detailed comparisons of the hypersonic wedge flow solutions yielded by the CUSP scheme and AUSM have been presented in Ref. [14]. The shock capturing capabilities of both schemes are essentially equal. A comparison of shock profiles for the exact and the simplified coefficients is given in Fig. 11. We have chosen the first-order scheme in order to address the pure shock capturing capability of the CUSP scheme without interference from the limiter. The simplified dissipation coefficients of Eq. (22) produce strong oscillations at the shock, even though there is substantial physical diffusion present. Hence it is concluded that an accurate implementation of dissipation coefficients is a must for hypersonic flows with strong shocks. 
Comparisons of computation times indicate that the CUSP scheme needs about $40 \%$ more computer time than the basic scalar dissipation. The MATD scheme only requires about $15 \%$ additional time. MATD requires less CPU time primarily because it needs only a single evaluation of the limiter function. Due to lower inherent dissipation, computations with the CUSP formulation converge somewhat slower for transonic flows than those with simple scalar dissipation. The major advantage of the CUSP approach is that it is more accurate and more robust than scalar viscosity. Our numerical tests indicate that the accuracy of the CUSP scheme is intermediate between scalar and matrix dissipation for transonic flows. For hypersonic flows it seems to be more robust than the matrix viscosity even though it is not TVD.

Since the CUSP scheme is implemented through artificial dissipative terms, it does not have to be applied at each stage of the Runge-Kutta method. In particular, the diffusive fluxes can be evaluated only at the first, third, and fifth stages of a five-stage method, just as is typically done for the scalar numerical dissipation.

\section{Concluding Remarks}

The CUSP scheme has been studied and analyzed. A detail comparison has been made between the CUSP, MATD, and scalar dissipation schemes. For transonic inviscid flows the CUSP scheme allows better resolution of shock waves, since they are captured with one interior point. However, the aerodynamic quantities such as lift and drag obtained with the CUSP scheme are not as accurate on coarser meshes (i.e., $320 \times 64$ cells or less) as those calculated with the MATD scheme. Both the CUSP and MATD formulations give high accuracy in the computation of high Re number flat-plate flow. For transonic viscous flows and coarser meshes the accuracy in aerodynamic coefficients is better with the MATD scheme than with the CUSP scheme. This loss in accuracy with the CUSP scheme on coarser grids appears to be a consequence of limiter activation (i.e., reduction to first order). Convergence of the HCUSP scheme has been improved by introducing the aspect-ratio scaling factor of Eq. (20).

With our present choice of HCUSP dissipation coefficients it has been shown that the resolution of strong shock waves occurring in hypersonic flows is possible whereas the simplified coefficients that were published previously failed.

The CUSP scheme requires roughly $40 \%$ more computer time than the scalar scheme, while the MATD scheme needs about $15 \%$ more time. Using only pressure in the switches for MATD rather than using a different measure for each equation reduces the robustness of the algorithm but requires less computer time. Convergence behavior with the CUSP and MATD schemes is similar. For hypersonic flows the CUSP scheme seems to be more robust than the MATD scheme.

\section{Acknowledgement}

We would like to thank Dr. Norbert Kroll for his help in understanding the CUSP formulas. 


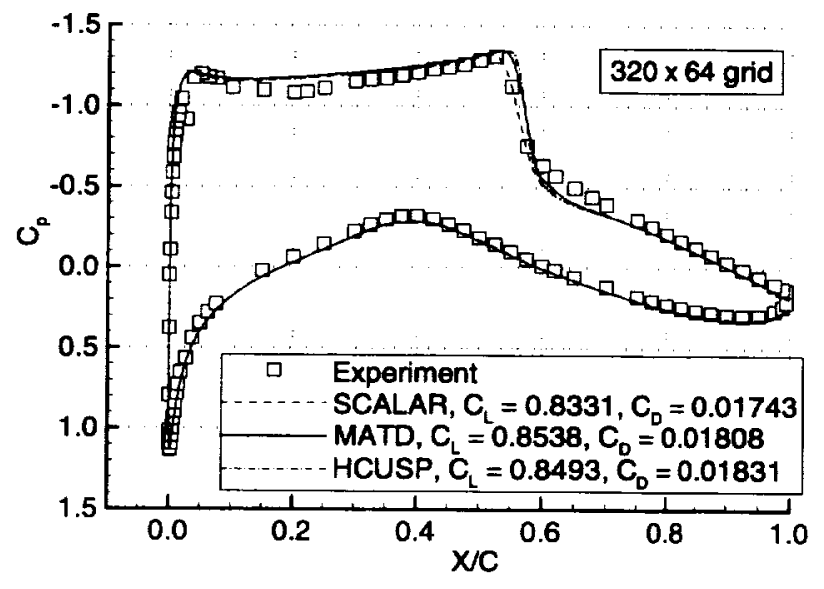

Figure 7a: Comparison of pressure distributions with SCALAR, MATD, and HCUSP schemes on $320 \times 64$ grid (RAE 2822 airfoil, $M=0.73, \alpha=2.79^{\circ}, R e=6.5 \times 10^{6}$ ).

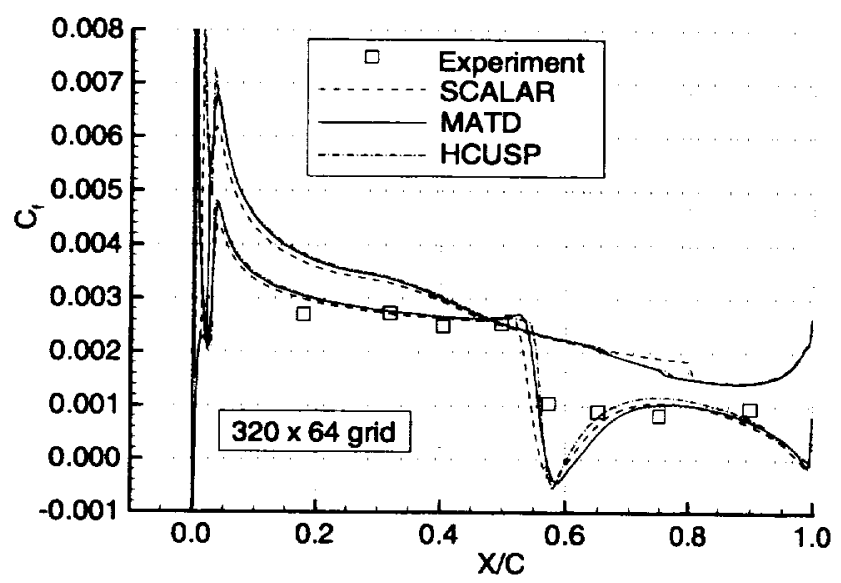

Figure 7b: Comparison of skin-friction distributions with SCALAR, MATD, and HCUSP schemes on $320 \times 64$ grid (RAE 2822 airfoil, $M=0.73, \alpha=2.79^{\circ}, R e=6.5 \times 10^{6}$ ). 


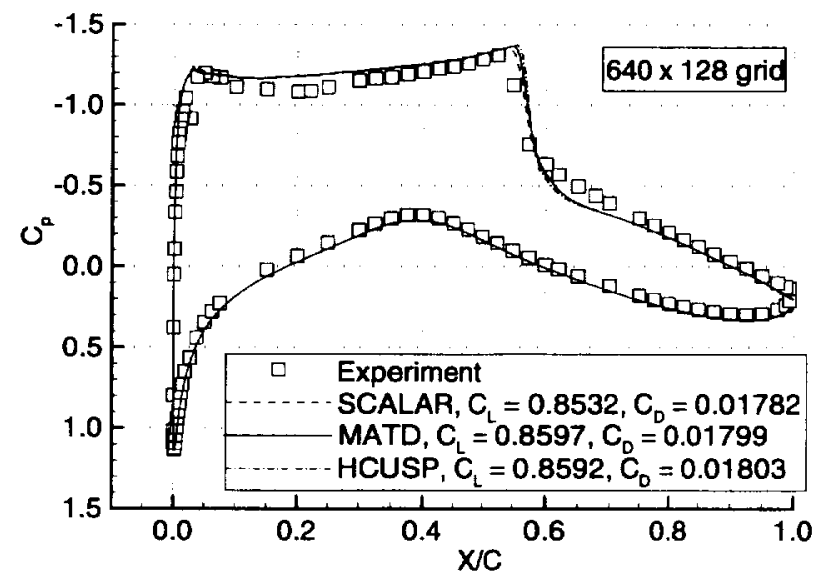

Figure 8a: Comparison of pressure distributions with SCALAR, MATD, and HCUSP schemes on $640 \times 128$ grid (RAE 2822 airfoil, $M=0.73, \alpha=2.79^{\circ}, R e=6.5 \times 10^{6}$ ).

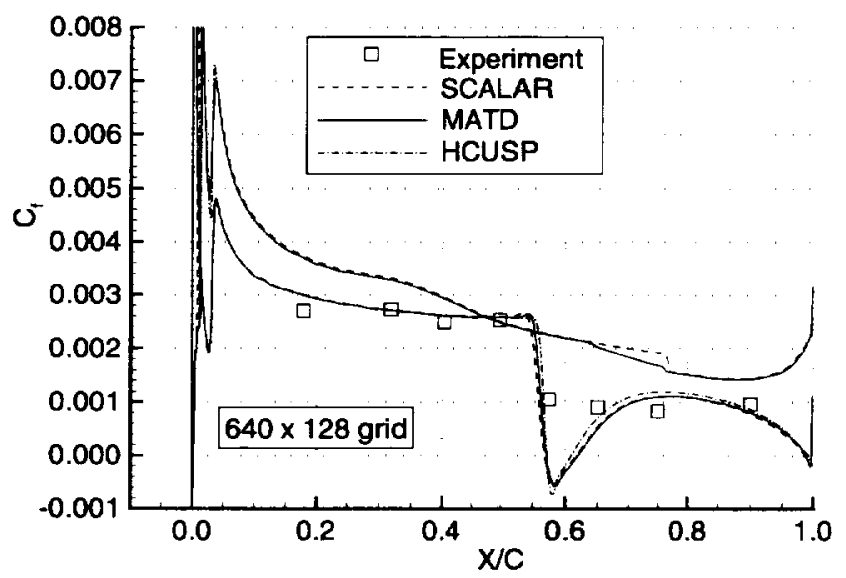

Figure 8b: Comparison of skin-friction distributions with SCALAR, MATD, and HCUSP schemes on $640 \times 128$ grid (RAE 2822 airfoil, $M=0.73, \alpha=2.79^{\circ}, R e=6.5 \times 10^{6}$ ). 


\begin{tabular}{|c|r|c|c|c|c|}
\hline $\begin{array}{c}\text { Dissipation } \\
\text { Scheme }\end{array}$ & Grid & $C_{L}$ & $C_{D}$ & $C_{D_{p}}$ & $C_{D_{f}}$ \\
\hline SCALAR & $160 \times 32$ & 0.8172 & 0.01728 & 0.01275 & 0.004532 \\
& $320 \times 64$ & 0.8331 & 0.01743 & 0.01194 & 0.005487 \\
& $640 \times 128$ & 0.8532 & 0.01782 & 0.01225 & 0.005574 \\
\hline MATD & $160 \times 32$ & 0.8304 & 0.01818 & 0.01251 & 0.005662 \\
& $320 \times 64$ & 0.8538 & 0.01808 & 0.01250 & 0.005571 \\
& $640 \times 128$ & 0.8597 & 0.01799 & 0.01246 & 0.005535 \\
& $1280 \times 256$ & 0.8611 & 0.01800 & 0.01246 & 0.005544 \\
\hline HCUSP & $160 \times 32$ & 0.7987 & 0.01926 & 0.01367 & 0.005594 \\
& $320 \times 64$ & 0.8493 & 0.01831 & 0.01263 & 0.005679 \\
& $640 \times 128$ & 0.8592 & 0.01803 & 0.01245 & 0.005585 \\
\hline
\end{tabular}

Table 1: Lift and drag coefficients for turbulent flow over RAE 2822 airfoil (RAE 2822, $M_{\infty}=0.73$, $\left.\alpha=2.79^{\circ}, R e_{c}=6.5 \times 10^{6}\right)$.

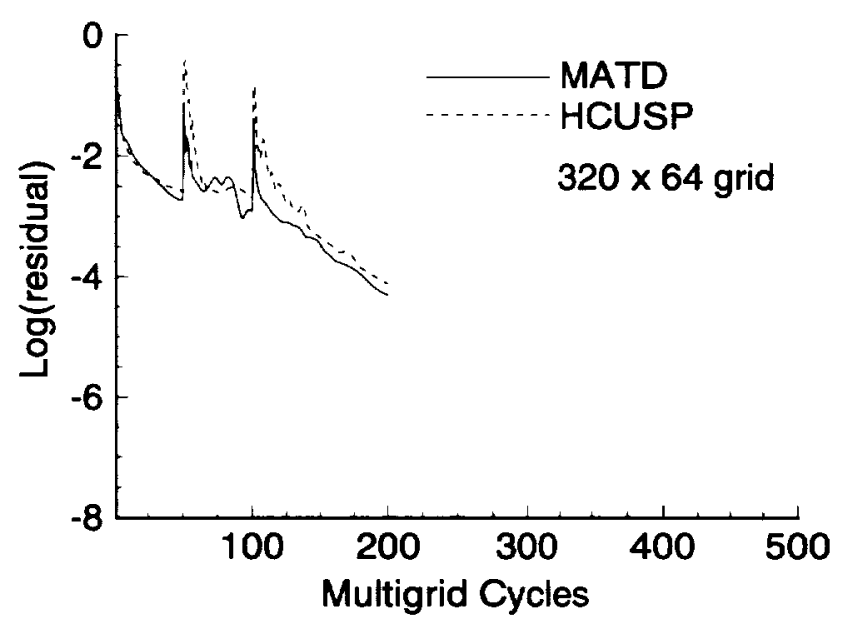

9a: MATD and HCUSP schemes.

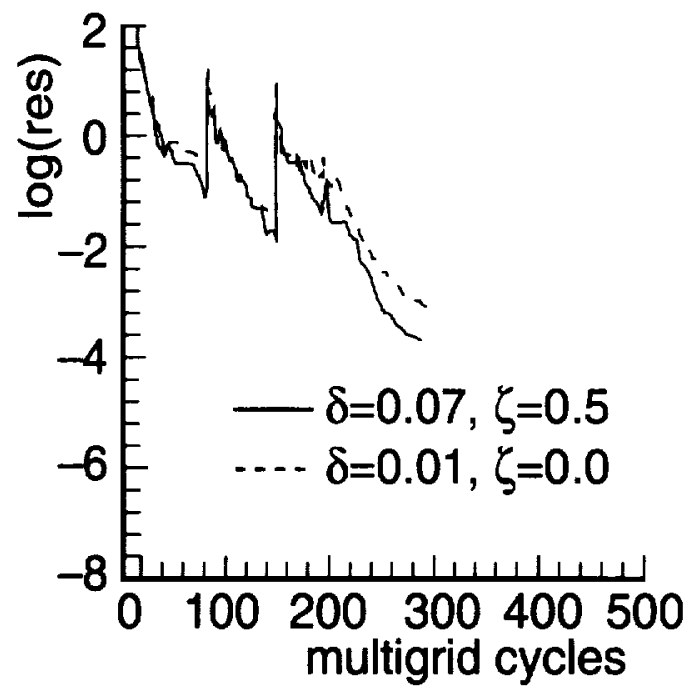

9b: Effect of modified $\nu c$ on HCUSP scheme.

Figure 9: Convergence histories for turbulent flow computations (RAE 2822 airfoil, $M_{\infty}=0.73$, $\alpha=2.79^{\circ}, R e_{c}=6.5 \times 10^{6}$ ). 

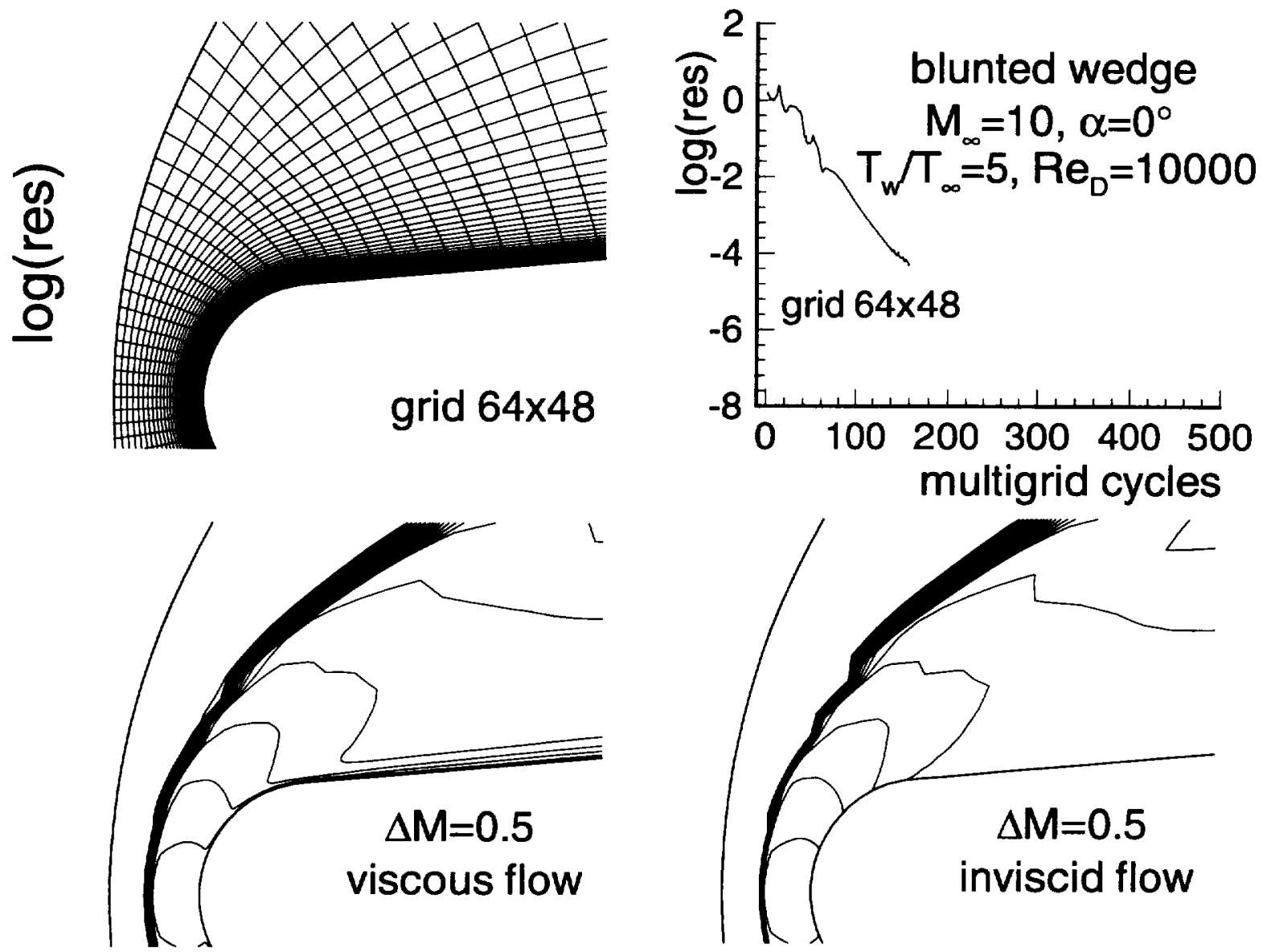

Figure 10: Viscous and inviscid hypersonic flow over 2-D wedge. 

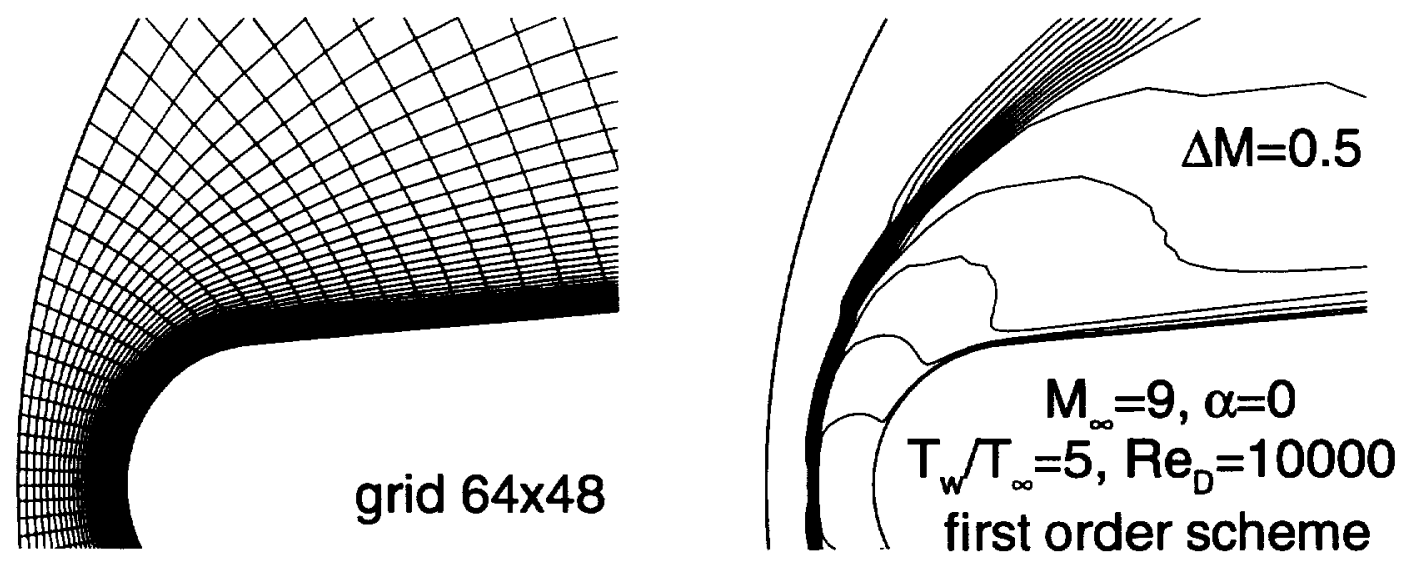

\section{center line flow}

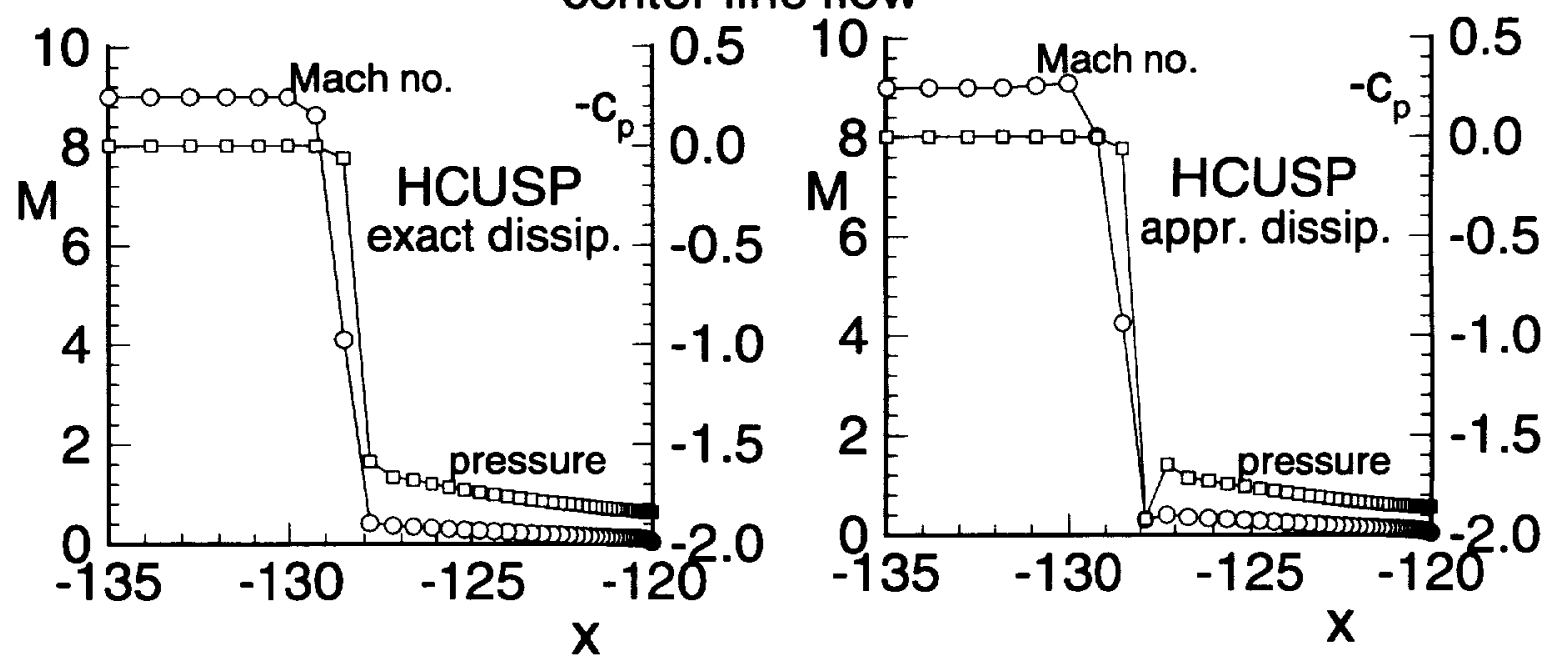

Figure 11: Influence of HCUSP dissipation coefficients on hypersonic flow over 2-D wedge. 


\section{References}

[1] Allmaras, S., Contamination of Laminar Boundary Layers by Artificial Dissipation in Navier-Stokes Solutions, Proc. Conf. Numerical Methods in Fluid Dynamics, 1992.

[2] Cook, P.H., McDonald, M.A., Firmin, M.C.P., AERFOIL RAE 2822 Pressure Distributions, and Boundary Layer and Wake Measurements, AGARD Advisory Report 138, 1979.

[3] Harten, A., High Resolution Schemes for Hyperbolic Conservation Laws, J. Comput. Physics, 49:357-393, 1983.

[4] Jameson, A., Schmidt, W., Turkel, E., Numerical Solutions of the Euler Equations by Finite Volume Methods Using Runge-Kutta Time-Stepping Schemes, AIAA Paper 81$1259,1981$.

[5] Jameson, A. Artificial Diffusion, Upwind Biasing, Limiters and their effect on Accuracy and Multigrid Convergence in Transonic and Hypersonic Flow, AIAA Paper 93-3559, 1993.

[6] Jameson, A., Analysis and Design of Numerical Schemes for Gas Dynamics I: Artificial Diffusion, Upwind Biasing, Limiters and their Effect on Accuracy and Multigrid Convergence, Inter. J. Comput. Fluid Dynamics, 4:171-218, 1995.

[7] Jameson, A., Analysis and Design of Numerical Schemes for Gas Dynamics II: Artificial Diffusion and Discrete Shock Structure, Inter. J. Comput. Fluid Dynamics, 5:1-38, 1995.

[8] Jameson, A., Positive Schemes and Shock Modelling for Compressible Flows, Inter. J. Numer. Methods Engineering, 20:743-776, 1995.

[9] Jiang, Y.T., Damodaran, M., Lee, K.H., High Resolution Finite Volume Calculation of Turbulent Transonic Flow Past Airfoils, AIAA-96-2377-CP.

[10] Liou, M.-S., Steffen, C.J., A New Flux Splitting Scheme, J. Comput. Physics, 107:23-39, 1993.

[11] Liou, M-S., A Sequel to AUSM: AUSM+, J. Comput. Physics, 129:364-382, 1996.

[12] Liou, M.-S., Wada, Y., A Quest Towards Ultimate Numerical Flux Schemes, CFD Review (Eds. M. M. Hafez and K. Oshima), 251-278, John Wiley and Sons, 1995.

[13] Martinelli, L., Jameson, A., Validation of a Multigrid Method for the Reynolds Averaged Equations, AIAA Paper 88-0414, 1988.

[14] Radespiel, R., Longo, J.M.A., Bruck, S., Schwamborn, D., Efficient Numerical Simulation of Complex 3D Flows with Large Contrast, AGARD-Symposium on "Progress and Challenges in CFD Methods and Algorithms", Seville, Spain, 2-5 October 1995.

[15] Radespiel, R., and Kroll, N., Accurate Flux Vector Splitting for Shocks and Shear Layers, J. Comput. Physics, 121:66-78, 1995.

[16] Radespiel, R., Swanson, R.C., Progress with Multigrid Schemes for Hypersonic Flow Problems, J. Comput. Physics, 116:103-122, 1995. 
[17] Roe, P. L., Approximate Riemann Solvers, Parameter Vectors and Difference Schemes, J. Comput. Physics, 43:357-372, 1981.

[18] Schlicting, H., Boundary Layer Theory, VII-th edition, McGraw-Hill, NY, 1979.

[19] Swanson, R.C., Turkel, E., Artificial Dissipation and Central Difference Schemes for the Euler and Navier-Stokes Equations, AIAA Paper 87-1107-CP, 1987.

[20] Swanson, R.C., Turkel, E., On Central Difference and Upwind Schemes, J. Comput. Physics, 101:292-306, 1992.

[21] Swanson, R.C., Turkel, E., Aspects of a High-Resolution Scheme for the Navier-Stokes Equations, AIAA Paper 93-3372-CP, 1993.

[22] Tatsumi, S., Martinelli, L. and Jameson, A Design, Implementation and Validation of Flux Limited Schemes for the Solution of the Compressible Navier-Stokes Equations, AIAA Paper 94-0647, 1994.

[23] Tatsumi, S., Martinelli, L., Jameson, A., A New High Resolution Scheme for Compressible Viscous Flow with Shocks, AIAA Paper 95-0466, 1995.

[24] Turkel, E., Vatsa, V.N., Radespiel, R., Preconditioning Methods for Low Speed Flow, AIAA Paper 96-2460-CP, 1996.

[25] Turkel, E., Radespiel, R., Kroll, N. Assessment of Two Preconditioning Methods for Aerodynamic Problems, to appear Computers and Fluids. 



\begin{tabular}{|c|l}
\hline REPORT DOCUMENTATION PAGE & $\begin{array}{l}\text { Form Approved } \\
\text { OMB No. 0704-0188 }\end{array}$ \\
\hline
\end{tabular}

Public reporting burden for this collection of information is estimated to average 1 hour per response, including the time for reviewing instructions, searching existing data sources. gathering and maintaining the data needed, and completing and reviewing the collection of information. Send comments regarding this burden estimate of any other aspect of this Davis Highway, Suite 1204, Arlington, VA 22202-4302, and to the Office of Management and Budget, Paperwork Reduction Project (0704-0188). Washington, DC 20503.

\begin{tabular}{|l|l|l}
\hline 1. AGENCY USE ONLY(Leave blank) & $\begin{array}{c}\text { 2. REPORT DATE } \\
\text { August } 1997\end{array}$ & $\begin{array}{l}\text { 3. REPORT TYPE AND DATES COVERED } \\
\text { Contractor Report }\end{array}$ \\
\hline
\end{tabular}

4. TITLE AND SUBTITLE

5. FUNDING NUMBERS

Comparison of Several Dissipation Algorithms for Central Difference Schenes

C NAS1-19480

WU 505-90-52-01

\section{AUTHOR(S)}

R. C. Swanson

R. Radespiel

E. Turkel

7. Performing organization name(S) and address(ES)

Institute for Computer Applications in Science and Engineering

Mail Stop 403, NASA Langley Research Center

Hampton, VA 23681-0001

8. PERFORMING ORGANIZATION REPORT NUMBER

ICASE Report No. $97-40$

9. SPONSORING/MONITORING AGENCY NAME(S) AND ADDRESS(ES)

National Aeronautics and Space Administration

Langley Research Center

Hampton, VA 23681-0001

10. SPONSORING/MONITORING AGENCY REPORT NUMBER

NASA CR-201726

ICASE Report No. $97-40$

\section{SUPPLEMENTARY NOTES}

Langley Technical Monitor: Dennis M. Bushnell

Final Report

AIAA 13th CFD Conference (June 27 - July 2, 1997)

12a. DISTRIBUTION/AVAILABILITY STATEMENT

Unclassified Unlimited

Subject Category 64

13. ABSTRACT (Maximum 200 words)

Several algorithms for introducing artificial dissipation into a central difference approximation to the Euler and Navier Stokes equations are considered. The focus of the paper is on the convective upwind and split pressure (CUSP) scheme, which is designed to support single interior point discrete shock waves. This scheme is analyzed and compared in cletail with scalar and matrix dissipation (MATD) schemes. Resolution capability is determined by solving subsonic, transonic, and hypersonic flow problems. A fuite-volume discretization and a multistage timestepping scheme with multigrid are used to compute solutions to the flow equations. Numerical results are also compared with either theoretical solutions or experimental data. For transonic airfoil flows the best accuracy on coarse meshes for aerodynamic coefficients is obtained with a simple MATD scheme.

14. SUBJECT TERMS

artificial viscosity, CUSP, SLIP

15. NUMBER OF PAGES 28

16. PRICE CODE $\mathrm{A} 03$

17. SECURITY CLASSIFICATION
OF REPORT
Unclassified

18. SECURITY CLASSIFICATION
OF THIS PAGE
Unclassified
19. SECURITY CLA
OF ABSTRACT

20. LIMITATION OF THIS PAG OF ABSTRACT 